\title{
Magnification Error in Digital Radiographs of the Cervical Spine Against Magnetic Resonance Imaging Measurements
}

\author{
Hideki Shigematsu ${ }^{1}$, Munehisa Koizumi ${ }^{2}$, Masana Yoneda ${ }^{1}$, Jin Iida ${ }^{2}$, Takuya Oshima ${ }^{1}$, Yasuhito Tanaka ${ }^{2}$ \\ ${ }^{1}$ Department of Orthopedic Surgery, Yamatotakada Municipal Hospital, Ymatotakada, Japan \\ ${ }^{2}$ Department of Orthopedic Surgery, Nara Medical University, Kashihara, Japan
}

\begin{abstract}
Study Design: Prospective study.
Purpose: The main purpose of this study was to clarify the range of magnification errors on digital plain radiographs and to determine if there is a correlation between the body mass index (BMI) of a patient and the magnification error.

Overview of Literature: Most clinicians currently use digital plain radiography. This new method allows one to access images and measure lengths and angles more easily than with the past technologies. In addition, conventional plain radiography has magnification errors. Although few articles mention magnification errors in regards to digital radiographs, they are known to have the same errors.

Methods: We used plain digital radiography and magnetic resonance imaging (MRI) to acquire images of the cervical spine with the goal of evaluating magnification errors by measuring the anteroposterior vertebral body lengths of $\mathrm{C} 2$ and $\mathrm{C} 5$. The magnification error (ME) was then calculated: $\mathrm{ME}=($ length on radiograph-length on MRI)/length on MRI $\times 100(\%)$. The correlation coefficient between the magnification error and BMl was obtained using Pearson's correlation analysis.

Results: Average magnification errors in C2 and C5 were approximately $18.5 \% \pm 5.4 \%$ (range, $0 \%-30 \%$ ) and $20.7 \% \pm 6.3 \%$ (range, $1 \%-32 \%)$. There was no positive correlation between $\mathrm{BMl}$ and the magnification error.

Conclusions: There were magnification errors on the digital plain radiographs, and they were different in each case. Maximum magnification error differences were 30\% (C2) and 31\% (C5). Based on these finding, clinicians must pay attention to magnification errors when measuring lengths using digital plain radiography.
\end{abstract}

Keywords: Cervical spine; Magnification error; Digital radiography; Body mass index; Picture archiving and communication system

\section{Introduction}

In the past, conventional hard-copy radiography images have been used. Currently, most clinicians (including ourselves) use digital plain radiography. This new method allows one to access images and measure lengths and angles more easily than with past technologies. The computer systems and programs used for this purpose are generically known as picture archiving and communication systems (PACSs).

Conventional plain radiographs have been reported to have some magnification errors [1]. Although few articles

Received May 30, 2012; Revised Jul 18, 2012; Accepted Aug 4, 2012

Corresponding author: Hideki Shigematsu

Department of Orthopedic Surgery, Yamatotakada Municipal Hospital,

1-1 Isonokitamachi, Yamatotakada 6358501, Nara, Japan

Tel: +81-745-53-2901, Fax: +81-745-53-2908, E-mail: shideki@naramed-u.ac.jp 
have mentioned magnification errors in regard to digital radiographs, they too exhibit these errors because digital radiography uses the same method to acquire photographs as conventional radiography. Clinicians may believe that digital radiography calculates the magnification error after uploading because of its simplicity, but this is not correct. When clinicians do not recognize this point, they may misjudge what is normal or abnormal. Spine surgeons frequently treat cervical spine diseases, such as dislocation and subluxation as well as spine trauma including fractures. As a guide, surgeons will use criteria based on distances measured on the radiograph. Because the cervical spine is comprised of a small proportion of bone tissue, a magnification error is an important consideration when making medical decisions.

The primary aim of this study was to assess the range of the magnification error on digital cervical plain radiographs. The secondary aim was to evaluate whether there was a correlation between the body mass index (BMI) of a patient and the degree of magnification error on digital plain radiographs.

\section{Materials and Methods}

We used PACS software (Centricity SEJ, GE Healthcare, Milwaukee, WI, USA) to access cervical digital radio- graphs (FCR Velocity, Fujifilm, Tokyo, Japan) and magnetic resonance imaging (MRI) scans (1.5T, SignaHDxt, GE Healthcare) of our outpatients to calculate the magnification error.

We recruited 54 patients ( 26 men, 28 women). We included only patients with cervical radiculopathy, cervical myelopathy, or mechanical neck pain who had underwent both digital radiography and MRI at the cervical level from January 2009 to August 2009 in our outpatient department. Our exclusion criteria included the presence of tumors, fractures, or infection in the cervical spine.

We chose C2 and C5 for assessment and measured the anteroposterior vertebral body length on the lateral cervical radiographs (Fig. 1A) [2]. In two of the patients, we were unable to confirm the presence of $\mathrm{C} 5$ clearly because of a short neck. The source beam was positioned a standard $150 \mathrm{~cm}$ away from the X-ray plate (Fig. 2). The midline T2-weighted sagittal images were used to assess the length (Fig. 1B). We were able to analyze C2 in 54 cases and $\mathrm{C} 5$ in 52 cases with digital plain radiography, and corresponding MRI was performed in these cases.

All measurements of length were conducted by the same researcher and were performed on two occasions on different days. Intraobserver agreement was calculated, and an average of the two measured values was obtained. To determine interobserver agreement, another doctor,
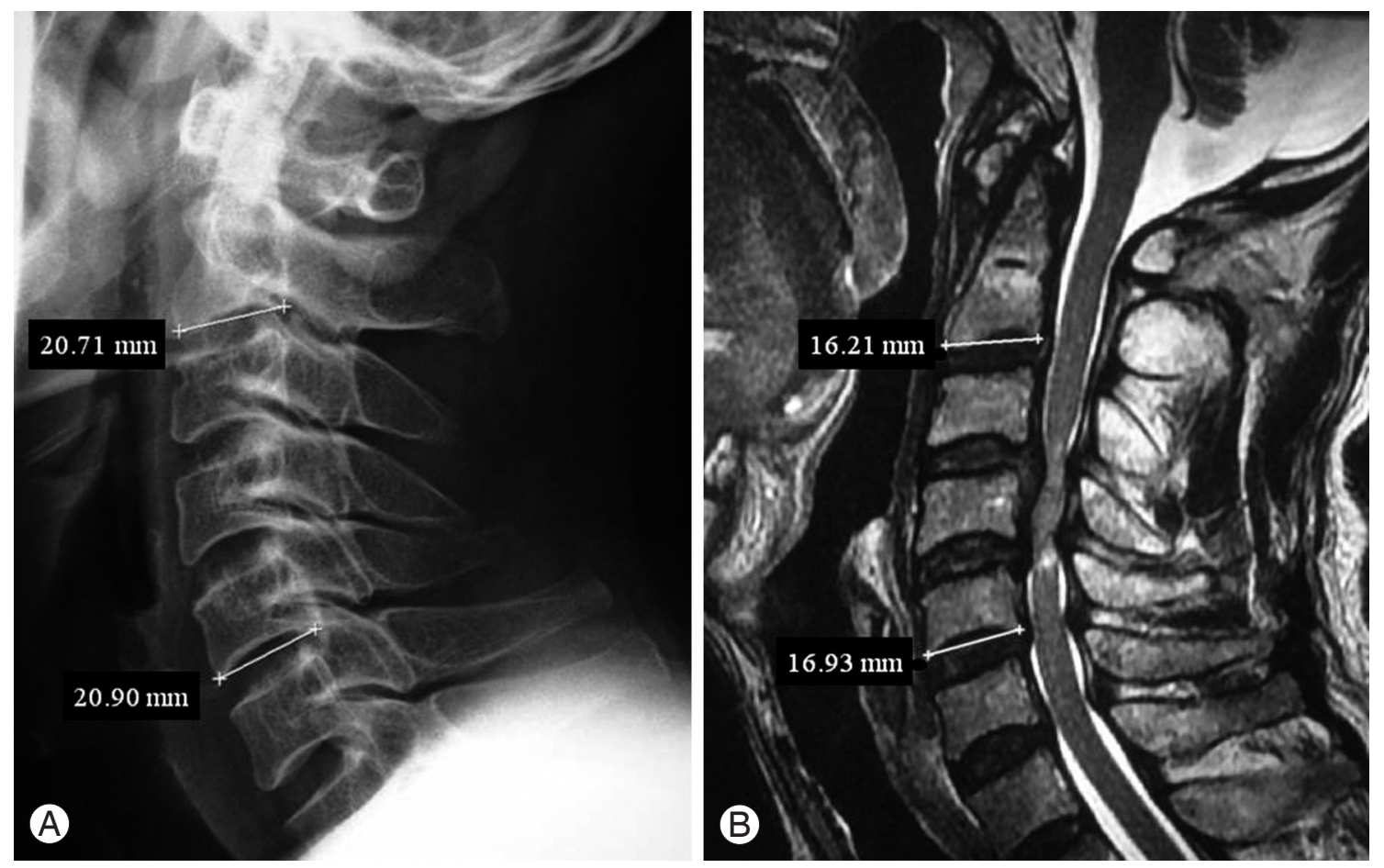

Fig. 1. (A) Measurements on the digital plain radiograph. (B) Measurements on magnetic resonance imaging. 
Table 1. Demographics of the subjects used in this study

\begin{tabular}{lcc} 
Characteristic & C2 & C5 \\
Sex (cases) & & \\
Male & 26 & 25 \\
\hline Female & 28 & 27 \\
Age (yr) & & \\
\hline Male & $61.1 \pm 13.3$ & $60.8 \pm 13.5$ \\
\hline Female & $55.6 \pm 15.7$ & $54.7 \pm 15.5$ \\
\hline Height (cm) & & \\
\hline Male & $165.3 \pm 5.6$ & $165.6 \pm 5.6$ \\
\hline Female & $156.7 \pm 8.2$ & $157.1 \pm 8.1$ \\
\hline Weight $(\mathrm{kg})$ & & \\
\hline Male & $65.0 \pm 9.7$ & $65.2 \pm 9.9$ \\
\hline Female & $54.7 \pm 8.2$ & $54.5 \pm 8.4$ \\
\hline Body mass index $\left(\mathrm{kg} / \mathrm{m}^{2}\right)$ & & $23.8 \pm 3.4$ \\
\hline Male & $23.8 \pm 3.3$ & $22.1 \pm 2.7$ \\
\hline Female & $22.2 \pm 2.8$ & \\
\hline
\end{tabular}

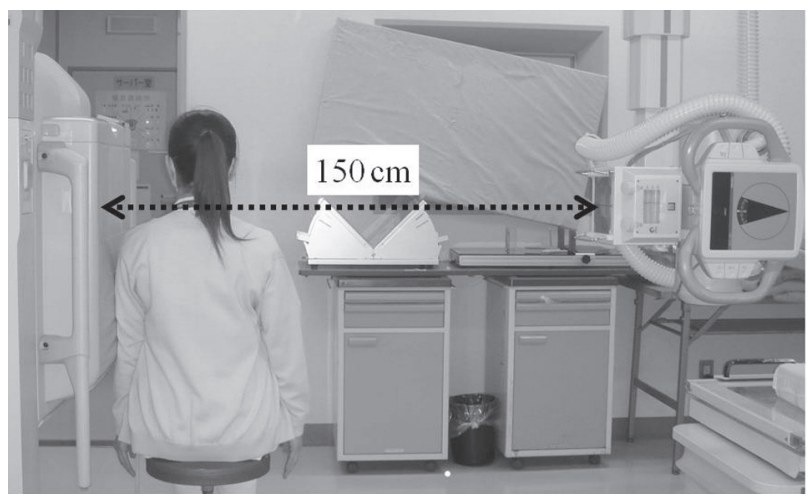

Fig. 2. Positioning the patient for photography.

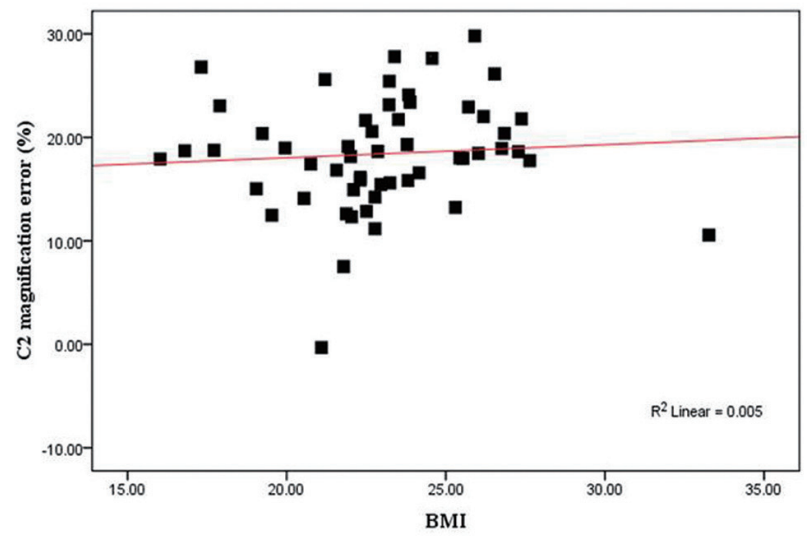

Fig. 3. Correlation between the magnification error and the patients' body mass index (BMI) values at C2. who was blinded to the patients' information, measured the same radiographs and MRI scans. The intraobserver and interobserver agreement for measurements were found to be excellent.

The interobserver error was as follows: radiography at C2: 0.967 (95\% confidence interval [CI], 0.943-0.981); radiography at C5: 0.970 (95\% CI, 0.948-0.983); MRI at C2: 0.958 (95\% CI, 0.928-0.976), MRI at C5: 0.928 (95\% CI, 0.874-0.959).

The intraobserver error was as follows: radiography at C2: 0.937 (95\% CI, 0.892-0.963), radiography at C5: 0.947 (95\% CI, 0.909-0.983); MRI at C2: 0.828 (95\% CI, 0.705-0.900), MRI at C5: 0.862 (95\% CI, 0.761-0.921).

The magnification error (ME) was calculated as follows:

$\mathrm{ME}=($ length on radiograph-length on $\mathrm{MRI}) /$ length on MRI×100 (\%).

Based on the BMI, we divided these patients into an underweight group $\left(<18.5 \mathrm{~kg} / \mathrm{m}^{2}\right)$ and an overweight group $\left(>25 \mathrm{~kg} / \mathrm{m}^{2}\right)$ for further assessment. The correlation coefficient between the magnification error and BMI was obtained using Pearson's correlation analysis. The Mann-Whitney's U-test was used to evaluate the difference between the underweight and overweight groups. $p<0.05$ was considered statistically significant.

Our institutional review board approved this study.

\section{Results}

The demographic data are as follows and are expressed

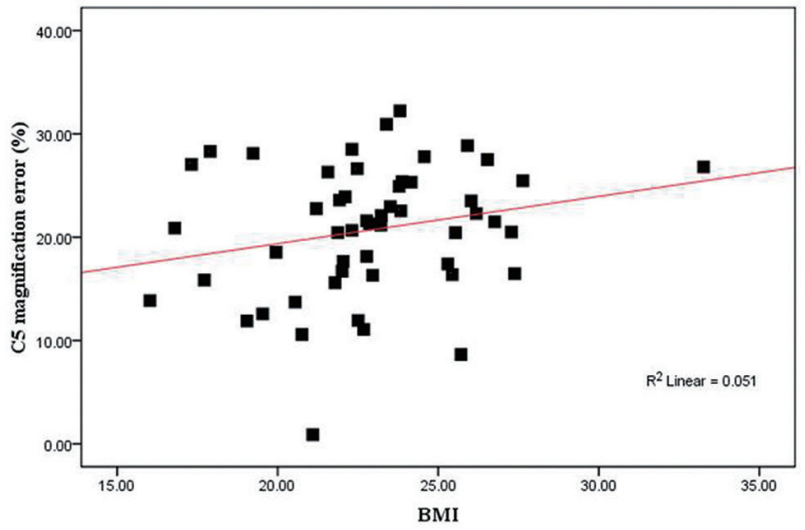

Fig. 4. Correlation between the magnification error and the patients' body mass index (BMI) at C5. 
Table 2. Effect of the subjects' body mass index on the magnification error

\begin{tabular}{lccc} 
Level & Body mass index & \% Magnification error & $p$-value \\
C2 & & & $18.5 \pm 5.4$ \\
\hline All $(n=54)$ & $23.0 \pm 3.1$ & $19.4 \pm 6.0$ & 0.60 \\
\hline Male $(n=26)$ & $23.8 \pm 3.3$ & $17.5 \pm 4.8$ & 0.75 \\
\hline Female $(n=28)$ & $22.2 \pm 2.8$ & & 0.40 \\
C5 & & $20.7 \pm 6.3$ & 0.11 \\
All $(n=52)$ & $22.9 \pm 3.1$ & $23.0 \pm 5.5$ & 0.70 \\
Male $(n=25)$ & $23.8 \pm 3.4$ & $18.6 \pm 6.4$ & 0.28 \\
\hline Female $(n=27)$ & $22.1 \pm 2.7$ & & \\
\hline
\end{tabular}

Table 3. Effect of being underweight or overweight on the magnification error

\begin{tabular}{|c|c|c|c|}
\hline Level & Underweight group (BMI<18.5) & Overweight group (BMI>25) & $p$-value \\
\hline $\mathrm{C} 2$ & $21.0 \pm 3.8$ & $19.8 \pm 4.8$ & 0.60 \\
\hline C5 & $21.2 \pm 6.5$ & $21.2 \pm 5.6$ & 0.99 \\
\hline
\end{tabular}

$\mathrm{BMI}$, body mass index.

as the averages \pm standard deviation (Table 1). 1) C2: 54 patients (26 men; age, $61.1 \pm 13.3$ years; BMI, $23.8 \pm 3.3 \mathrm{~kg}$ / $\mathrm{m}^{2}$; 28 women; age; $55.6 \pm 15.7$ years; BMI, $22.2 \pm 2.8 \mathrm{~kg} /$ $\mathrm{m}^{2}$ ). 2) C5: 52 patients (25 men; age, $60.8 \pm 13.5$ years; BMI, $23.8 \pm 3.4 \mathrm{~kg} / \mathrm{m}^{2}$; 27 women; age, $54.7 \pm 15.5$ years; BMI, $22.1 \pm 2.7 \mathrm{~kg} / \mathrm{m}^{2}$ ).

The ages of the patients ranged from 25 to 86 years (average, $58.2 \pm 14.8$ years).

The overall radiography measurements were 19.2 \pm 1.9 $\mathrm{mm}$ (range, $15.1-24.3 \mathrm{~mm}$ ) at $\mathrm{C} 2$ and $21.6 \pm 3.3 \mathrm{~mm}$ (range, $14.8-32.4 \mathrm{~mm}$ ) at C5. The overall MRI measurements were $16.2 \pm 1.5 \mathrm{~mm}$ (range, $13.4-22.0 \mathrm{~mm}$ ) at $\mathrm{C} 2$ and $17.8 \pm 2.4 \mathrm{~mm}$ (range, $13.1-25.9 \mathrm{~mm}$ ) at C5.

The magnification results are summarized in Table 2. The average magnification errors in $\mathrm{C} 2$ and $\mathrm{C} 5$ were approximately $18.5 \% \pm 5.4 \%$ (range, $0 \%-30 \%$ ) and $20.7 \% \pm 6.3 \%$ (range, $1 \%-32 \%$ ). There was no significant difference between the magnification errors in C2 and C5 ( $p=0.08$, Mann-Whitney U-test), and there was no significant correlation between BMI and the magnification error (C2: $r=0.07, p=0.60$; C5: $r=0.23, p=0.11$ ) (Figs. 3, 4). We analyzed the data for the sexes but found no significant correlation between BMI and magnification error.

We divided the cases based on our definition of the BMI values $\left(\mathrm{kg} / \mathrm{m}^{2}\right)$ : $<18.5$, underweight group; $>25.0$, overweight group). The underweight group was comprised of five patients examined at $\mathrm{C} 2$ and five at $\mathrm{C} 5$. The overweight group was comprised of 14 patients examined at $\mathrm{C} 2$ and 13 at C5. In the underweight group, the magnification error at C2 and C5 was $21.0 \% \pm 3.8 \%$ and $21.2 \% \pm 6.5 \%$, respectively. In the overweight group, the magnification error at C2 and C5 was $19.8 \% \pm 4.8 \%$ and $21.2 \% \pm 5.6 \%$. There was no significant difference in the magnification errors between the two groups (Table 3 ).

\section{Discussion}

In this study, we evaluated the magnification errors on digital plain radiographs. The errors varied among the different cases $(0 \%-32 \%)$ (Figs. 3, 4); however, a significant correlation between the patients' BMIs and the degree of the magnification errors was not observed. Also, there was no significant difference in the magnification errors between the underweight and overweight groups.

A number of previous studies have examined the magnification error on pelvic radiographs [3-6]. For accurate preoperative planning of total hip replacement, it is important to know and take into account the magnification error. Few studies have examined the magnification error on digital films at the cervical spine. Spine surgeons usually use radiographs for diagnosis and/or assessment. For example, atlantoaxial subluxation is diagnosed in patients with an atlantodental interval $>3 \mathrm{~mm}$ [7], and subaxial subluxation is diagnosed when there is $>2 \mathrm{~mm}$ displace- 
ment [8]. Because it is important that we still use the unit of length, not a ratio, when addressing the cervical spine, we thought that it would be meaningful to know the degree of magnification error that occurs.

Theoretically, the magnification error is determined based on the distance between the film and the spine [6], which depends on maintaining the distance from the film and not altering the focus. That is, if the object moves away from the film, the magnification error increases. Ravi and Rampersaud [2] reported that the magnification error was correlated with the patient's BMI. Based on their study, the distance between the film and the spine might increase as the BMI increases.

We did not find a significant correlation between the patients' BMIs and the magnification errors. There are some possible reasons for this lack of correlation. First, the BMIs of our patients ranged between $16.0 \mathrm{~kg} / \mathrm{m}^{2}$ and $33.3 \mathrm{~kg} / \mathrm{m}^{2}$, and within this range there might not be significant differences in the distance between the film and the cervical spine. Second, although we determined that the distance of the film to the source of the beam was 150 $\mathrm{cm}$ without exception for the cervical spine, clinically there was a possibility that the distance was different for each patient because the radiology technician usually obtained numerous radiographs in the outpatient department and might not have been able to maintain the precise distance. Although we cannot determine precisely why these individual differences occurred, we believe the important consideration is that digital plain radiography always has a magnification error, which is similar to hardcopy radiography, and there is great variability among patients.

Routine use of computed tomography (CT) and MRI for diagnostic purposes has resulted in more widespread radiation exposure. It has also put a greater demand on the radiology department, with increased costs. Plain radiography is inexpensive, and we can evaluate dynamic factors from flexion and/or extension views-points that are advantageous when compared with CT or MRI. Consequently, measurements from plain radiographs cannot be completely eliminated by assessing cervical spine disorders.

Our study has some limitations. First, we did not include patients with a high BMI (e.g., BMI, $40-50 \mathrm{~kg} / \mathrm{m}^{2}$ ), so we could not evaluate magnification errors of highBMI patients. Second, we did not directly evaluate the correlation between BMI and the distance from the film to the spine. Third, we used MRI for the alternative measurement. Although measurements taken from CT images are accurate to within $1 \mathrm{~mm}$ of the actual size [9], a previous study showed that MRI is as accurate as CT [2].

Even with these limitations, we clarified the difference in magnification errors among patients. Again, we believe that the most important point is that there are magnification errors to consider when using digital plain radiography and that these errors vary widely among patients. Although there are many clinical scenarios where linear measurements from plain radiographs are used to assist in diagnostic and/or clinical decision-making, clinicians need to consider and compensate for magnification error. We also recommend evaluating cervical spine digital radiographs using an angle or a ratio (e.g., percent slip in the lumbar spine), neither of which is influenced by the magnification error.

\section{Conclusions}

In this study, we identified magnification errors when using digital plain radiography, and their degree varied among patients. There was no significant correlation between BMI and the magnification error. Finally, when using digital plain radiography for measurements, the magnification error should be measured and taken into consideration.

\section{Conflict of Interest}

No potential conflict of interest relevant to this article was reported.

\section{References}

1. Heller JG, Viroslav S, Hudson T. Jefferson fractures: the role of magnification artifact in assessing transverse ligament integrity. J Spinal Disord 1993;6:3926.

2. Ravi B, Rampersaud R. Clinical magnification error in lateral spinal digital radiographs. Spine (Phila $\mathrm{Pa}$ 1976) 2008;33:E311-6.

3. Wimsey S, Pickard R, Shaw G. Accurate scaling of digital radiographs of the pelvis. A prospective trial of two methods. J Bone Joint Surg Br 2006;88:150812.

4. Heinert G, Hendricks J, Loeffler MD. Digital templat- 
ing in hip replacement with and without radiological markers. J Bone Joint Surg Br 2009;91:459-62.

5. King RJ, Makrides P, Gill JA, Karthikeyan S, Krikler SJ, Griffin DR. A novel method of accurately calculating the radiological magnification of the hip. J Bone Joint Surg Br 2009;91:1217-22.

6. Franken M, Grimm B, Heyligers I. A comparison of four systems for calibration when templating for total hip replacement with digital radiography. J Bone Joint Surg Br 2010;92:136-41.

7. Pellicci PM, Ranawat CS, Tsairis P, Bryan WJ. A prospective study of the progression of rheumatoid arthritis of the cervical spine. J Bone Joint Surg Am 1981;63:342-50.

8. Yurube T, Sumi M, Nishida K, et al. Progression of cervical spine instabilities in rheumatoid arthritis: a prospective cohort study of outpatients over 5 years. Spine (Phila Pa 1976) 2011;36:647-53.

9. Raja YR, Fehlings MG, James SH, et al. Validation of digital radiology measurement tools for quantitative spinal imaging. Top Spinal Cord Inj Rehabil 2006;12:11-21. 